\title{
Transcontinentales
}

Sociétés, idéologies, système mondial

\section{Religion et pouvoir d'État en Asie centrale}

Un système fragilisé

Religion and State Power in Central Asia. A Fragilised System

\section{Sébastien Peyrouse}

\section{(2) OpenEdition}

Journals

Édition électronique

URL : http://journals.openedition.org/transcontinentales/282

DOI : $10.4000 /$ transcontinentales.282

ISBN : 978-2-8218-1410-3

ISSN : $1775-397 X$

\section{Éditeur}

Editions de la maison des sciences de l'homme

\section{Édition imprimée}

Date de publication : 31 décembre 2005

Pagination : 69-84

ISBN : 2200-92168-3

ISSN : $1950-1684$

\section{Référence électronique}

Sébastien Peyrouse, «Religion et pouvoir d'État en Asie centrale», Transcontinentales [En ligne], 1 | 2005, document 5, mis en ligne le 03 février 2011, consulté le 08 septembre 2020. URL : http:// journals.openedition.org/transcontinentales/282; DOI : https://doi.org/10.4000/transcontinentales. 282

Ce document a été généré automatiquement le 8 septembre 2020.

Tous droits réservés 


\title{
Religion et pouvoir d'État en Asie centrale
}

\author{
Un système fragilisé \\ Religion and State Power in Central Asia. A Fragilised System
}

\section{Sébastien Peyrouse}

1 Après la chute de l'URSS et l'indépendance acquise en 1991, les pouvoirs des pays d'Asie centrale (Kazakhstan, Kirghizstan, Ouzbékistan, Tadjikistan, Turkménistan), en place depuis la période soviétique, ont cherché à s'adapter aux changements en cours. Ils sont alors passés sans difficulté du discours communiste à celui du nationalisme, vantant les mérites d'une indépendance supposée chèrement acquise. Très attentifs au regard occidental porté sur eux, ils se mirent à la recherche de nouveaux référents idéologiques leur permettant l'intégration sur la scène internationale: démocratie, économie de marché et respect des droits de l'homme font aujourd'hui pleinement partie des stéréotypes à savoir manier pour obtenir des soutiens financiers de pays donateurs et d'organismes internationaux ${ }^{1}$. Chaque État a déclaré suivre sa propre voie vers l'économie de marché. Alors que le Kazakhstan et le Kirghizstan ont entamé, dès le début des années 1990, des réformes brutales sur le modèle russe de la "thérapie de choc » en privatisant largement leur économie, l'Ouzbékistan et le Turkménistan ont opté pour une évolution mesurée, le plus souvent proche du statu quo ante. Le Tadjikistan, soumis à plus de quatre années de guerre civile (1992-1996), tente lui aussi, désormais, de s'engager sur la voie des réformes qu'il avait très brièvement entamées au lendemain de l'indépendance ${ }^{2}$.

2 Après les attentats du 11 septembre 2001, les cinq républiques, aux confins de l'Iran, de l'Afghanistan et de la Chine, se sont soudain trouvées placées sous un regard médiatique international bien souvent porté sans recul historique. Les interrogations posées quant à l'avenir de l'islam dans cette région et à l'expansion éventuelle de ses petits groupes islamistes militants ont trop souvent masqué une question plus structurelle : comment les régimes post-soviétiques, très marqués par l'autoritarisme, ont-ils géré la question religieuse après l'indépendance? Cette question spécifique renvoie à d'autres interrogations qui, elles, ne sont pas propres à l'Asie centrale : celle 
des rapports entre État et société dans des régimes non-démocratiques, du rôle des milieux religieux officiels comme étouffoir, régulateur ou porte-voix des insatisfactions sociales, celle enfin de la recherche de stratégies préservant la stabilité. En effet, dans chacun des États d'Asie centrale, hormis peut-être le Kirghizstan "postrévolutionnaire » de 2005, le pouvoir se présente aujourd'hui comme le seul garant de la stabilité. Il a élaboré au fil des années une schématisation outrancière de toute forme de non-conformisme intellectuel, social ou religieux, en l'assimilant à une opposition systématiquement qualifiée d'intégriste.

3 Le prisme religieux semble à cet égard particulièrement pertinent pour réfléchir à la question de la réforme et à ses enjeux dans des pays de tradition musulmane officiellement laïcs ${ }^{3}$. Analyser ici la gestion du fait religieux par les pouvoirs postsoviétiques d'Asie centrale illustre la difficulté à réformer des États qui conjuguent aujourd'hui plusieurs handicaps : la sortie malaisée de régimes de tradition autoritaire, une tiers-mondisation économique et sociale qui paupérise rapidement la majeure partie de la population (le Kazakhstan étant à part à cet égard), enfin, les interrogations portées à l'encontre d'une religion majoritaire, l'islam, que les États de la région et une grande part de la communauté internationale, États-Unis en tête, redoutent de voir dériver vers des mouvances extrémistes, aux connections transnationales, et cherchant à déstabiliser les pouvoirs en place. L'objet de cet article n'est donc pas d'étudier les mouvances islamistes, mais ce qui pourrait être pour elles un terreau fertile: l'autoritarisme actuel du pouvoir politique et la soumission des instances de l'islam officiel à celui-ci. C'est en effet de l'aptitude de l'islam officiel à servir de régulateur aux revendications de certaines couches de la population, mais surtout de la capacité du pouvoir à ne pas liquider toute opposition laïque qui le laisserait dans un face-à-face avec l'islamisme, que se dessinera le succès ou l'échec de cette minorité d'activistes religieux.

\section{État des lieux : le post-soviétisme en question?}

L'Asie centrale d'aujourd'hui reste avant tout un espace post-soviétique, héritier d'un passé colonial russe de plus d'un siècle et demi. Si un certain nombre de réformes ont été engagées, les quinze années écoulées depuis 1991 ont été de toute évidence insuffisantes pour se défaire du système politico-économique qui a prévalu pendant plusieurs décennies et ce, quelle que soit la volonté de réformes, affichée ou réelle, des chefs d'État et de leurs gouvernements ${ }^{4}$. La continuité avec le régime soviétique est avant tout politique: l'État a très largement préservé le principe d'un fort autoritarisme, pratiquant une ingérence idéologique permanente. 
Figure 1

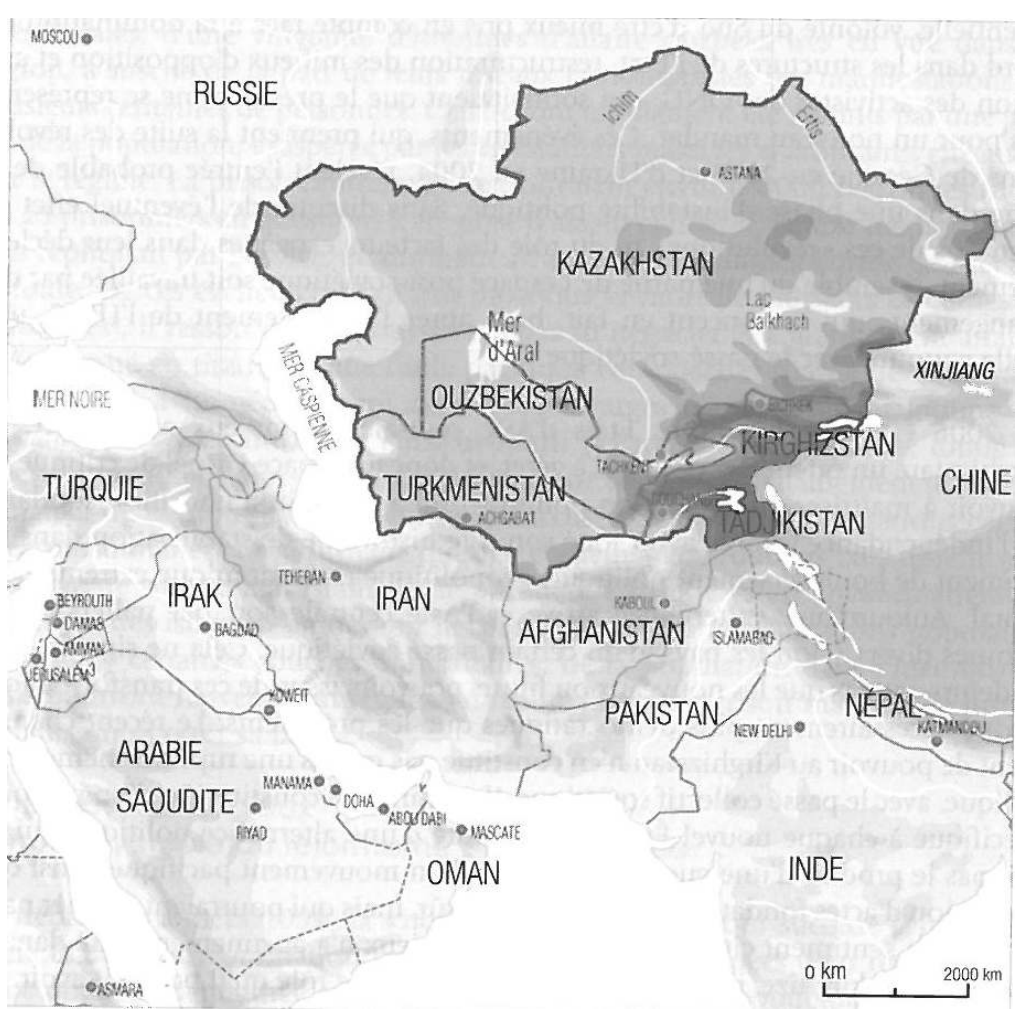

L'Asie centrale dans son contexte régional

5 L'expression et l'organisation de la société civile sont souvent extrêmement restreintes. L'Ouzbékistan et le Turkménistan n'octroient pas la moindre liberté d'expression tandis que les trois autres républiques, qui avaient offert au lendemain de l'indépendance davantage d'espace démocratique, ont aujourd'hui elles aussi très fortement restreint le champ des libertés. Les principaux journaux d'opposition ont été fermés et les opposants politiques sont soumis à de rudes pressions, emprisonnés ou contraints à l'exil pour échapper à la répression gouvernementale. Le Tadjikistan qui, au terme des accords de paix de 1997, avait dû garantir de réels espaces de liberté de parole et d'engagement politique, tente lui aussi aujourd'hui de réduire la marge de manœuvre de ses opposants.

6 Plus d'une décennie après la rupture de 1991, l'Asie centrale est aujourd'hui à un tournant de son histoire. En mars 2005, les fraudes relevées durant les élections législatives au Kirghizstan conduisent à des mouvements de masse dans le Sud de la république, qui se propagent ensuite à la capitale, Bichkek. En quelques jours, le pouvoir est renversé, à la surprise de tous, et le président Askar Akaev abandonne son poste sans résistance. Cette "révolution", que certains prévoyaient pour les élections présidentielles d'octobre 2005, s'explique par différents facteurs: mécontentement populaire récurrent à l'encontre du népotisme de la famille présidentielle, volonté du Sud d'être mieux pris en compte face à la domination du Nord dans les structures de l'État, restructuration des milieux d'opposition et agitation des activistes des ONG, qui souhaitaient que le président ne se représente pas pour un nouveau mandat. Ces événements, qui prennent la suite des révolutions de Géorgie en 2003 et d'Ukraine en 2004, révèlent l'entrée probable de la zone dans une phase d'instabilité politique. Sans discuter de l'éventuel effet de dominos de ces « révolutions» ni du rôle des facteurs 
exogènes dans leur déclenchement, il semble qu'une partie de l'espace post-soviétique soit travaillée par des changements qui annoncent en fait, bien après l'effondrement de l'URSS, une réelle coupure avec le passé soviétique.

7 En 2006 et 2007, les autres États d'Asie centrale auront eux aussi, après le Kirghizstan, un calendrier électoral à gérer, et donc un espace virtuel de critique du pouvoir à maitriser. La génération politique qui a conduit la transition soudaine de l'indépendance semble avoir joué son rôle historique de stabilisation dans un moment de bouleversement politique, géopolitique et économique extrêmement brutal. Aujourd'hui, l'alternance arrive et l'Asie centrale sort par paliers, à des rythmes divers selon les pays, d'un certain passé soviétique. Cela ne signifie bien évidemment pas que les nouveaux ou futurs pouvoirs issus de ces transformations seront nécessairement plus démocratiques que les précédents. Le récent changement de pouvoir au Kirghizstan n'en constitue pas moins une rupture, même symbolique, avec le passé collectif soviétique. Il confirme la constitution d'un parcours spécifique à chaque nouvel État, la possibilité d'une alternance politique qui ne soit pas le produit d'une guerre civile mais d'un mouvement pacifique, ainsi que la création d'actes fondateurs, mythifiés bien sûr, mais qui pourraient donner naissance à un sentiment citoyen. Si le facteur religieux n'a aucunement joué dans la « révolution » kirghize, il n'en est pas de même dans le rôle qu'il pourrait avoir, ou qu'il se voit attribuer, en Ouzbékistan.

En effet, l'Ouzbékistan, "homme malade» de la région, connaît une phase de durcissement politique et de crispation sociale importante, perceptible depuis les attentats du 16 février 1999, puis accentuée par à-coups au fil d'événements internationaux (11 septembre 2001), régionaux (incursions islamistes au Tadjikistan et au Kirghizstan à l'été 1999 et 2000, renversement du président kirghize A. Akaev en mars 2005) et nationaux (vague d'attentats suicides en mars et juillet 2004, certains ayant été perpétrés, pour la première fois en Asie centrale, par des femmes). En ayant éliminé depuis des années toute opposition laïque, les autorités ouzbèkes se sont enfermées dans un face-à-face avec les islamistes. Bien qu'interdits et clandestins, ceux-ci poursuivent leur critique politique du régime et sont les seuls, à l'heure actuelle, à proposer dans leurs tracts et leurs samizdats des alternatives à l'absolutisme du président Islam Karimov. Pour autant, les risques de déstabilisation de l'Ouzbékistan ne sont pas nécessairement liés à la seule question religieuse: jouent aussi les difficultés quotidiennes grandissantes, les luttes d'influence entre groupes économicopolitiques, et la question très sensible de la justice sociale, à l'origine de l'insurrection du 13 mai 2005 dans la ville d'Andijan, dans la vallée du Ferghana.

En effet, l'emprisonnement, dû à un retournement d'alliance de la part des autorités locales, d'une vingtaine d'hommes d'affaire ouzbeks très en vue dans la région, a suscité de la part de leurs réseaux factionnalistes des manifestations de plusieurs centaines de personnes. Ceux-ci ont rapidement été rejoints par une partie de la population, exaspérée par les arrestations massives et arbitraires effectuées par le régime. La prison centrale, où se trouvaient environ 4000 personnes (dont les 23 prisonniers en question) a été prise d'assaut et libérée. Le soir même, le pouvoir répliquait par la force, en donnant à l'armée et à la milice l'ordre de tirer sur la foule. De ces événements qui ont provoqué la mort de plusieurs centaines de personnes ${ }^{5}$, il ressort que le pouvoir n'a pas su négocier et a grandement affaibli sa légitimité en tirant sur une foule pacifique composée en partie de femmes et d'enfants. Il a immédiatement accusé les insurgés d'appartenir au groupe dit Akkramiyya, soi-disant 
lié au Hizb ut-Tahrir («Parti de la libération », fondé en Palestine en 1954), alors que le fait religieux ne constituait aucunement le vecteur principal de la protestation, même si les accusés géraient des associations caritatives musulmanes. En affirmant que les événements d'Andijan constituent une tentative de coup d'État islamiste, le président Karimov valide involontairement les efforts des islamistes à devenir les porte-parole des revendications populaires et renforce certaines couches, minoritaires, de la population dans l'idée qu'eux seuls pourront renverser un régime en réalité honni pour son manque de justice sociale et non pour sa laïcité.

\section{Le renouveau religieux comme symbole du réformisme post-soviétique}

Au début des années 1990, la «nouvelle Asie centrale » qui succède à près d'un siècle et demi de domination russe, dont soixante-dix années d'un régime soviétique officiellement athée, ouvre une ère d'espoir pour la population croyante de la région, très majoritairement musulmane. Les hommes au pouvoir appartiennent à la population éponyme ${ }^{6}$ et redonnent à l'islam, au moins en apparence, ses lettres de noblesse. La construction de nombreux édifices de culte, la nouvelle visibilité des pratiques et un intérêt officialisé pour toute spiritualité sont présentés comme un "élan» de la population vers la religion. De nombreux courants missionnaires, musulmans comme chrétiens, voient dans ce retournement historique l'avènement d'une ère nouvelle et s'installent alors en Asie centrale. Ces mesures publiques en faveur de la liberté religieuse sont d'autant plus nécessaires que les présidents en place cherchent à se défaire de leur image pesante d'anciens apparatchiks soviétiques, tous formés dans le moule du Parti communiste de leur république. Les pouvoirs politiques sont également en quête, dans le cadre de la construction de nouveaux États-Nations, d'une légitimité symbolique qu'ils élaborent au travers d'une histoire revisitée par les historiographes officiels. La religion apparaît à ce titre comme l'un des éléments mobilisateurs de l'identité nationale.

Les discours des présidents en place évoquent ainsi sans ambages le rôle fondateur de la religion dans la construction de la société nouvelle et lui vouent une fonction normative des valeurs morales : "il est indispensable de prendre tout ce qu'il y a de mieux dans la religion, car l'essentiel, c'est que la religion soutienne la morale $»^{7}$ affirmait ainsi le président kirghize Askar Akaev, démis en mars 2005. La religion, autrefois à la périphérie de la vie publique, en serait donc devenue, à en croire les chefs d'État, une composante majeure. Selon le président ouzbek Islam Karimov, «aucune société ne peut imaginer son avenir sans le développement du potentiel spirituel, des valeurs spirituelles et morales dans la conscience de sa population. $»^{8}$ Cette mise en valeur rhétorique de la religion permet aux pouvoirs d'accréditer l'idée qu'ils offrent à l'expression religieuse, et tout particulièrement à l'islam, une place inédite dans l'histoire récente de la région, après la domination tsariste, considérée comme chrétienne orthodoxe, puis l'athéisme soviétique.

12 L'islam réapparaît au grand jour par la construction et la réouverture, partout en Asie centrale, de milliers de mosquées. L'éducation religieuse, réprimée sous le régime soviétique, est autorisée, tandis qu'une grande partie des mollahs sort de l'ombre. Si le régime soviétique avait laissé un certain nombre d'entre eux agir dans la mesure où ils acceptaient de se conformer au cadre d'expression imparti, beaucoup avaient préféré 
se réfugier dans la clandestinité ou mener leurs activités religieuses dans la plus grande discrétion. En 1992 toutefois, personne ne craint plus de détenir des ouvrages religieux à son domicile et un individu peut se revendiquer pratiquant sans pour autant encourir de conséquences professionnelles. Sont également impliquées dans cette résurgence apparente de la foi toutes les croyances minoritaires, en particulier le christianisme, seconde religion de la région compte tenu des importantes minorités européennes qui y sont présentes depuis le $\mathrm{XIX}^{\mathrm{e}}$ siècle. Des églises orthodoxes, mais également des maisons de prière catholiques et protestantes sont reconstruites et/ou rouvertes 9 .

Chaque Constitution, dans son préambule ou ses premiers articles, jette les bases de la laïcité dans le cadre d'un État présenté comme démocratique ${ }^{10}$ et stipule la séparation de la religion et de l'État ${ }^{11}$. Deuxième principe fondamental, chaque État garantit la liberté de culte, la possibilité de célébrer une religion ou de n'en pratiquer aucune ${ }^{12}$. L'athéisme d'État est interdit et toutes les religions bénéficient officiellement de droits similaires ${ }^{13}$. Chaque individu est égal devant la loi quelle que soit son appartenance religieuse, et ne peut être discriminé en raison de la religion qu'il pratique, ni contraint de préciser son appartenance confessionnelle ${ }^{14}$. La croyance est abordée sous le terme générique de "religion", écartant ainsi toute hiérarchisation officielle entre confessions. Aucune Constitution en Asie centrale ne fait donc de l'islam la religion officielle du pays, la séparation de la religion et de l'État étant systématiquement spécifiée ; aucun texte fondateur ne contient même les termes d'islam, de musulman ou de Coran et nul statut spécifique n'est imposé aux non-musulmans. Ainsi, les législations des pays d'Asie centrale ne répondent pas aux modèles en vigueur dans nombre de pays du Proche et Moyen-Orient. Les révisions constitutionnelles menées au Kazakhstan en 1995 et au Kirghizstan en 2003 ne modifient en rien ces fondements laïcs, auxquels les pouvoirs comme les sociétés centrasiatiques sont attachés dans leur très grande majorité.

\section{Limites des réformes et maintien du cadre de contrôle soviétique}

Le « renouveau » du fait religieux en Asie centrale post-soviétique trouve cependant rapidement ses limites. La "résurgence» religieuse n'est pas, en effet, sans conséquences pour les pouvoirs politiques, qui assument de moins en moins les risques selon eux encourus à laisser se développer des mouvances religieuses dont quelquesunes sont extrémistes (Mouvement islamique d'Ouzbékistan et Hizb ut-Tahrir). Ce danger est d'autant plus vivement appréhendé que le prétendu "vide » idéologique post-soviétique est supposé conduire une partie de la population vers le militantisme politico-religieux, perçu comme une alternative aux autorités politiques en place. Les États d'Asie centrale sont donc confrontés à de multiples interrogations et à des prises de décisions riches en contradictions. Comment préserver un cadre laïc, effacer le souvenir de l'athéisme militant et promouvoir la religion dans ses diverses confessions ? Les pouvoirs en place tentent de répondre à ces questions dès la première moitié des années 1990 par un très net tournant autoritaire, et arguent alors de leur devoir de faire face aux «menaces de déstabilisation politique et sociale» dont les groupes religieux extrémistes seraient en grande partie responsables.

15 La liberté religieuse est donc appréhendée au même titre que les libertés politiques: sans pour autant invoquer un retour aux normes soviétiques, qui restent condamnées 
dans le discours officiel, les autorités insistent sur la nécessité de maintenir un contrôle gouvernemental sur les activités religieuses et donc de limiter l'espace d'expression publique de la foi. Les instances politiques locales considèrent en réalité, sans l'affirmer ouvertement sur la scène internationale, que le schéma occidental n'est pas applicable en Asie centrale : la liberté religieuse est une menace pour la stabilité d'États jeunes, dont les peuples sont déclarés comme non encore prêts pour la démocratie. Sont ici moins visées les confessions chrétiennes, qui ne nourrissent aucune ambition politique, que les mouvements islamiques fondamentalistes. Ceux-ci sont présentés par les pouvoirs centrasiatiques comme un phénomène essentiellement étranger, émanant d'États tels que l'Afghanistan, le Pakistan ou les pays arabes, et très rarement assumés comme des phénomènes ayant également des fondements internes à chaque pays.

Gérer le fait religieux suppose de choisir les mouvements et confessions que le pouvoir considère comme officiels, adaptés à l'histoire et à la culture nationales, et ceux qu'il condamne comme étrangers. Cette classification est particulièrement révélatrice puisqu'elle est très largement calquée sur celle qui avait prévalu sous le régime soviétique, elle-même en partie héritée de l'époque tsariste. Les autorités définissent ainsi des religions dites " traditionnelles », considérées comme étant la " foi nationale » d'un groupe ethnique. Pour les cinq républiques d'Asie centrale, sont reconnus en tout premier lieu l'islam sunnite hanéfite dans sa version imposée par les autorités politiques et représentée officiellement par les Directions spirituelles des musulmans, et le christianisme russe orthodoxe en tant que religion de la principale minorité nationale de la région. Les représentants de ces deux religions sont systématiquement invités lors des cérémonies officielles et même impliqués dans certaines instances étatiques (par exemple l'Assemblée des peuples au Kazakhstan) et ce, malgré la séparation de la religion et de l'État spécifiée dans les textes constitutionnels. Les autres confessions «nationales » bénéficient d'un statut limité à la pratique cultuelle de leur minorité: Église arménienne, Église uniate pour les Ukrainiens, Église luthérienne pour les Allemands, Église catholique pour les Polonais, judaïsme.

17 Les autres mouvements religieux sont définis comme "non traditionnels " et très régulièrement classés dans l'ensemble compact des "sectes", ce qui permet au pouvoir, comme sous le régime soviétique, de faire peser sur eux une épée de Damoclès. Ces mouvements sont en effet tolérés s'ils acceptent de se plier au cadre, souvent restreint, qui leur est imposé, et sont réprimés dans le cas contraire. Ce qualificatif de sectaire permet de distinguer non seulement les mouvements chrétiens, qui dérangent les pouvoirs en place par leur prosélytisme envers les représentants des nationalités de tradition musulmane (essentiellement les mouvements protestants missionnaires, très actifs dans la région), mais également toutes les tendances de l'islam qui ne répondent pas aux normes de l'État. Ainsi, le caractère discriminant du terme de "secte » est utilisé à des fins politiques tant dans la propagande athée que dans celle des nouveaux pouvoirs, rendant le classement à géométrie variable : l'antisoviétisme dont ils étaient accusés a aujourd'hui laissé place à d'autres termes faisant eux aussi office de condamnation officielle, comme le "wahhabisme", auquel sont systématiquement associées les idées de déstabilisation, de fanatisme, d'intégrisme et de terrorisme.

Les États d'Asie centrale tentent donc par leur autoritarisme de s'ériger en protecteurs de la stabilité et de la concorde sociale contre ces mouvements : prétexter la mise en danger de la stabilité républicaine et laïque constitue leur argument central. Ce leitmotiv n'est pas spécifique à la zone, mais s'inscrit dans un cadre largement post- 
soviétique : plusieurs autres républiques de l'ex-URSSont également instrumentalisé la menace de l'islam intégriste afin de museler l'opposition et de justifier certaines politiques répressives, par exemple les deux guerres en Tchétchénie menées par le pouvoir russe. Le legs soviétique reste donc essentiel, en matière religieuse comme dans d'autres domaines. Les États se sentent mal assurés face au pouvoir, présumé ou réel, des religions ; leur volonté de laïcité est en fait un mode de protection des élites au pouvoir face à un islam qui pourrait se révéler déstabilisateur pour les privilèges acquis ; la question nationale constitue aujourd'hui encore l'élément clé de la définition d'un groupe et permet de justifier la reconnaissance ou non d'une religion historiquement liée à lui. Par ailleurs, l'approche par les pouvoirs politiques des mouvements religieux reste procédurière et fondée sur des vexations administratives quotidiennes.

\section{Les conséquences : surveiller et punir en Asie centrale}

19 Dans chacun des cinq États de la région, l'ensemble du fait religieux est aujourd'hui géré et contrôlé par une structure étatique, les Conseils aux affaires religieuses, créés sous le régime soviétique. Certains gouvernements actuels ont essayé de se disculper des accusations de perpétuer le système soviétique en remplaçant ces Conseils par des structures analogues ou en changeant simplement leur nom ${ }^{15}$. Ces institutions, qui avaient vocation sur l'ensemble de la région sous le régime précédent, n'exercent désormais leurs compétences qu'à l'échelle de chacune des républiques d'Asie centrale. Elles constituent à ce titre l'un des principaux instruments d'encadrement et de contrôle des institutions religieuses et des pratiquants, et restent largement sous l'influence des autorités politiques, en particulier au Turkménistan et en Ouzbékistan, de plus en plus marqués par le culte de la personnalité présidentielle. Les responsables placés à leur tête sont systématiquement des proches du pouvoir, généralement des personnes de confiance du président lui-même. Comme à l'époque soviétique, les Conseils sont officiellement chargés d'instaurer un dialogue entre l'État et les mouvements religieux. Ils n'en conservent pas moins leurs prérogatives de contrôle, parfois très étroit, de toute expression religieuse, avec toutefois de très sensibles différences entre les républiques. Le Kazakhstan et le Kirghizstan laissent en effet une marge de manœuvre certaine aux divers courants religieux, non seulement musulmans et orthodoxes, mais également aux confessions protestantes ou même à celles qui ne se réclament ni de l'islam et du christianisme (par exemple Hare Krishna), alors que ces confessions sont très étroitement contrôlées en Ouzbékistan et interdites au Turkménistan.

Chaque État dispose également d'une Direction spirituelle ou d'une structure équivalente chargée de superviser plus spécifiquement la communauté musulmane et les oulémas. Tous présentent cette institution comme un moyen de faciliter et d'encourager le développement de la vie religieuse. Ce principe est cependant contesté par certains, puisque aucune institution suprême n'est censée exister en islam: les Directions spirituelles apparaissent alors comme des institutions émanant de la domination russo-soviétique, qui plus est stalinienne, et dénuées de toute légitimité théologique. Aujourd'hui comme hier, l'idée de non-ingérence est donc appliquée de manière asymétrique, au détriment des religions dans leur rapport à la sphère publique, et en faveur de l'État, qui dispose d'un droit de regard sur elles. Malgré ces 
contestations, les autorités politiques n'en ont pas moins atteint, en partie, leur objectif, c'est-à-dire régir une grande partie du fait religieux tout en affirmant l'indépendance de la religion. Ce contrôle reste malgré tout partiel puisque les pouvoirs ne parviennent pas à éradiquer les mouvances fondamentalistes comme le Mouvement islamique d'Ouzbékistan ou le Hizb ut-Tahrir.

21 L'une des premières fonctions des Conseils aux affaires religieuses est d'enregistrer, c'est-à-dire d'accréditer, les communautés religieuses, faute de quoi elles ne peuvent exister légalement. Les gouvernements exercent par ce biais un étroit contrôle sur tout mouvement religieux qui souhaite s'installer sur leur territoire. L'enregistrement permet par ailleurs d'adapter la législation aux exigences locales et/ou circonstancielles : comme sous le régime soviétique, la procédure reste opaque et la décision est souvent laissée à la discrétion des instances compétentes ou aux pouvoirs dérogatoires des gouvernements. Ainsi, certains mouvements qui ne remplissaient pas les conditions requises ont été malgré tout enregistrés. D'autres, pourtant parvenus à satisfaire à toutes les exigences, ont été refusés au Turkménistan et en Ouzbékistan. La liberté d'enseignement religieux, encore en vigueur au Kazakhstan et au Kirghizstan, s'est trouvée de plus en plus limitée dans les trois autres républiques. Les restrictions pesant sur la littérature religieuse ne sont pas sans rappeler, elles aussi, les pratiques dont ont été victimes les croyants pendant les soixante-dix années de régime soviétique.

Ainsi, en Ouzbékistan et au Turkménistan, on constate aujourd'hui un net très recul des acquis de l'époque gorbatchévienne, la censure sur les ouvrages religieux étant systématique. Dans ces deux républiques, les sermons du vendredi font l'objet de toute l'attention des autorités et sont très strictement contrôlés. Alors qu'en 1993, 6000 mosquées étaient recensées en Ouzbékistan, leur nombre est tombé à 1967 en $1998^{16}$ et a continué de baisser dans les années suivantes. Le pays a également mené, ces dernières années, une répression systématique en arrêtant, emprisonnant et souvent en torturant tout individu soupçonné de pratiquer sa religion de manière trop zélée, et donc supposé être un intégriste potentiel. Les disparitions sont devenues fréquentes et les organisations de défense de droit de l'homme estiment qu'entre 5000 et 7000 prisonniers politiques sont détenus dans les prisons et les camps ouzbeks, la plupart pour des motifs religieux ${ }^{17}$.

\section{Le double jeu du pouvoir envers la question religieuse}

Les pouvoirs centrasiatiques actuels reconnaissent et mettent en exergue un certain nombre d'éléments religieux élevés au rang de marqueurs identitaires et étatiques. Ceux-ci sont toutefois systématiquement subordonnés aux desseins de l'État et au culte de la figure présidentielle. En invitant les mouvements religieux à constituer l'un des fondements du « renouveau national », les autorités contraignent ces mouvements à une "folklorisation" qui ne fait, là aussi, que perpétuer un processus largement entamé sous le régime soviétique. En effet, à partir de la Seconde Guerre mondiale, la réhabilitation du nationalisme russe par Staline conduit à une folklorisation des cultures de chaque république. Ce processus atteint son apogée sous Brejnev : les héros nationaux de chaque entité fédérée sont réinsérés dans un cadre soviétique tandis que les expressions populaires de la culture sont glorifiées au détriment de la "haute " littérature ou du passé politique. Les principales religions pratiquées en URSSsont elles 
aussi contraintes de se plier à ce strict cadre folklorique, et nombre de leurs représentants s'en accommodent en échange d'une certaine liberté de culte. Hier comme aujourd'hui, la majorité de la population exprime son sentiment religieux en participant à des jubilés, des journées de la culture, des fêtes traditionnelles et aux principaux rites de passage, sans pour autant fréquenter régulièrement les lieux de culte. Pour les minorités nationales, l'expression publique d'une culture religieuse a constitué et constitue encore un des modes d'affirmation identitaire. Les indépendances de 1991 n'ont donc fait qu'accélérer la «nationalisation » du religieux commencée depuis plus d'un demi-siècle.

Impliquer la religion dans un certain nombre de manifestations officielles et donner des droits aux principaux mouvements religieux permet également au pouvoir d'éroder la contestation. Cette tactique avait fait ses preuves en URSS, où une partie des croyants se déclarait satisfaite de sa liberté religieuse limitée. Aujourd'hui également, nombre d'oulémas se contentent des conditions actuelles dans la mesure où ils peuvent pratiquer leur culte, même dans un cadre restreint et soumis à certains choix idéologiques. Ce discours est récurrent au sein de l'Église orthodoxe, nul hiérarque ne se plaignant de pressions de la part des autorités. Dans toutes les républiques d'Asie centrale, les oulémas tout comme les grandes figures du clergé orthodoxe clament la liberté accordée et la juste gestion du religieux par le politique ${ }^{18}$. Le paroxysme est atteint au Turkménistan, où les mosquées et les églises deviennent autant des lieux de célébration du président Saparmurad Niazov et de sa politique que des lieux de culte. Les responsables musulmans et orthodoxes sont contraints de chanter les louanges du chef de l'État lors des services religieux. Cette politique a pour effet de marginaliser les quelques voix discordantes et d'empêcher leur accès au champ public. Elle va de pair avec une répression plus ou moins brutale.

Cette instrumentalisation est d'autant plus bénéfique aux pouvoirs en place que ceux-ci jouent sur les deux tableaux, mettant en avant leur reconnaissance de la religion tout en discréditant, au moins partiellement, les mouvements religieux impliqués. Ce processus est particulièrement illustré par le Parti de la renaissance islamique tadjik (PRIT), fer de lance de l'opposition au cours de la guerre civile au Tadjikistan et aujourd'hui encore seul parti islamiste officiellement reconnu dans toute l'Asie centrale ${ }^{19}$. Une fois intégré dans le paysage politique et devenu membre de la coalition gouvernementale, le parti a très largement modéré son discours et mis en sourdine son attente d'une islamisation de l'État ${ }^{20}$. Le pouvoir tadjik accède ainsi à la demande d'une partie de la population en faisant acte de son respect pour la religion, tout en affaiblissant alors les revendications et la fonction tribunitienne de l'islamisme. La modération actuelle du PRIT n'empêche toutefois pas l'opposition de se recomposer par le biais d'autres tendances politiques plus marginales et plus radicales dans leur volonté d'islamiser les structures étatiques.

\section{Les possibles réponses du champ religieux à la situation politique}

Dans toute la région, l'étatisation des institutions censées représenter «l'opinion publique musulmane » se double d'un processus de "retraditionalisation » de l'islam très largement encouragé par le pouvoir. Les autorités espèrent dissocier un «bon » islam national des mouvances salafistes internationalisées ou des courants islamistes 
politisés. À ce titre, les pouvoirs laïcs tout comme les Directions spirituelles ne cessent d'officialiser le culte des saints et de se référer au soufisme, présenté comme la forme la plus authentique de l'islam post-soviétique : il est apprécié pour ses préoccupations exclusivement spirituelles, son rejet de la politisation et même de l'engagement social, son aspect national et traditionnel. ${ }^{21}$

Les pouvoirs s'appuient pour cela sur le « clergé » musulman local, assuré que celui-ci s'efforcera d'encourager cette retraditionalisation, et jouent la carte du soutien à un certain conservatisme de mœurs en échange d'une dépolitisation. Les mollahs des petites mosquées de quartier, grâce auxquels l'islam a pu survivre comme élément de la vie sociale et culturelle à la période soviétique, sont en réalité des notables locaux fidèles aux choix effectués par leur clan ou leur communauté de quartier (mahalla). Ils revendiquent fortement les traditions spécifiques de leur pratique de l'islam, sont en faveur d'une réislamisation conservatrice apolitique et s'opposent à de nouvelles générations urbaines, plus cultivées et qui cherchent à réhabiliter une conception universaliste de l'islam. Le traditionalisme peut en effet être appréhendé comme un gage du respect de la séparation de l'État et de la religion : l'islam, minoritaire au sein d'un Empire tsariste orthodoxe puis illégitime au sein d'une Union soviétique athée, est aujourd'hui, dans son immense majorité, prêt à reconnaître l'existence d'un État laïc post-soviétique tant que sa liberté confessionnelle est respectée.

Les pouvoirs s'inquiètent toutefois de l'arrivée sur scène de nouvelles générations de théologiens, souvent formées à l'étranger ou dans les toutes jeunes institutions locales d'enseignement islamique, pour qui une islamisation en profondeur des sociétés postsoviétiques constituerait, sur le moyen terme, l'une des seules garanties de valorisation de leurs convictions idéologiques et de leur formation professionnelle. Par ailleurs, le " clergé » tient lui-même une position ambiguë dans sa propre instrumentalisation. La laïcité n'est pas nécessairement comprise par tous les théologiens musulmans comme étant positive en soi, en tant que garantie de l'autonomie de la foi, puisque l'islam se pense comme une réalité tout aussi bien religieuse que séculière. La participation du "clergé » musulman à sa propre étatisation se révèle ainsi ambiguë car certains espèrent y voir un mode d'entrisme dans les structures officielles et une discrète islamisation de l'État. Il semble toutefois qu'au contraire, ce soit celui-ci qui réussisse à séculariser les représentants de l'islam, devenus des fonctionnaires comme les autres.

Outre cet islam officiel, le champ politique centrasiatique est également investi, de manière dissidente, par les courants islamistes. Plusieurs groupes marginaux, tous interdits, revendiquent en effet aujourd'hui un retour aux normes premières de l'islam, la restauration du califat et un respect strict de la charia. La majorité d'entre eux ne relèvent toutefois pas du djihadisme internationalisé, mais peuvent plutôt être classés dans la catégorie islamo-nationaliste. Le Mouvement islamique d'Ouzbékistan, probablement le plus internationalisé puisque bénéficiant du soutien taliban, s'est trouvé grandement affaibli par la disparition de sa base arrière afghane à la fin de l'année $2001^{22}$. Le Parti de la renaissance islamique Tadjike a quant à lui toujours donné priorité à la solidarité ethnique sur l'idéologie. Il respecte aujourd'hui le cadre national, se fait le héraut du nationalisme tadjik contre l'Ouzbékistan et ne s'est jamais allié aux islamistes ouzbeks.

Le principal parti islamiste en Asie centrale, jamais légalisé, le Hizb ut-Tahrir, dont les membres sont majoritairement ouzbeks, développe lui aussi une argumentation avant tout nationale: il exige la démission du président Islam Karimov, la libération des 
prisonniers politiques, l'assouplissement des législations, etc. ${ }^{23}$ Il ne joue que peu la carte de la rhétorique internationaliste du califat mondial - ce qui ne signifie naturellement pas l'absence de liens avec des mouvances étrangères, en particulier sur le plan de l'aide financière - mais confirme que la détresse sociale et politique que connaît tout particulièrement l'Ouzbékistan fait le lit de ce fondamentalisme: la féminisation des attentats-suicides de 2004 et le choix pour leurs cibles de lieux symboliques du pouvoir ouzbek s'inscrivent dans des logiques plus nationales qu'internationales. L'insurrection du 13 mai 2005 à Andijan rappelle elle aussi combien l'enjeu réel des secousses que connaît le pays ne relève pas de stratégies religieuses mais de situations de crise sociale : l'extrême restriction de toute expression publique oblige une partie de la population à remettre en cause par la violence ce qu'elle considère être l'illégitimité du pouvoir actuel.

Le « post-islamisme » en tant que courant considérant que l'État n'est plus l'objectif à atteindre pour réislamiser la société est donc peu répandu dans l'espace postsoviétique; l'islamisme classique est lui aussi limité face à la très large domination, chez les théologiens musulmans de la région, d'un conservatisme apolitique à la recherche de la seule retraditionalisation de la société. L'interdiction de toute opposition laïque, en particulier en Ouzbékistan, ouvre toutefois le champ des revendications sociales aux mouvements islamistes, qui sont alors les seuls à encore " parler politique » et à émettre une opinion sur la gestion actuelle du pays. Cela ne suffit toutefois pas à présupposer qu'ils disposent réellement d'une représentativité quantifiable conséquente et il est peu probable qu'en cas d'élections démocratiques, les salafistes ouzbeks et tadjiks atteindraient des scores importants : la majorité de la population musulmane de l'ex-URSS reste en faveur d'un État laïc respectueux des droits religieux de chacun. L'enjeu n'est donc pas d'inviter à la reconnaissance juridique de ces mouvements islamistes en tant que partis politiques ni d'espérer leur acceptation du jeu démocratique, comme semblent le penser certaines institutions internationales comme l'OSCE ${ }^{24}$, mais de diversifier le paysage politique.

\section{Conclusion}

Comme nous avons cherché à le montrer ici, l'idée d'un " renouveau » religieux permis par l'effondrement du régime athée soviétique n'est pas sans ambiguïé, car elle masque en partie les réalités de l'Asie centrale contemporaine. La promotion du concept de « renouveau » a permis aux États apparus en 1991 d'affirmer qu'ils avaient donné naissance à une démocratie et rendu la liberté religieuse après l'effondrement du régime totalitaire. Ils ont en réalité empêché l'établissement d'un dialogue libre entre religion et société. L'idée de "renouveau " jette un voile sur les mesures de répression à l'encontre de l'islam, tout particulièrement en Ouzbékistan et au Turkménistan : le nombre de mosquées en fonction aujourd'hui, s'il est très largement supérieur à celui du régime soviétique, est bien moindre que dans les premières années de l'indépendance, le durcissement progressif du pouvoir face au fait religieux ayant eu raison de l'effervescence du début des années 1990.

Les changements proclamés en matière religieuse ont donc été et restent pour les régimes en place un moyen de mettre en avant leur prétendue volonté de réformes, même si la gestion du religieux apparaît en réalité comme l'un des témoignages essentiels de leur incapacité à penser dans un cadre conceptuel novateur le rapport 
entre État et religion. Le discours officiel sur le haut degré de réformisme des républiques cache en effet les innombrables mesures de répression politique et sociale qui affectent les cinq Républiques, et qui conduisent à une situation des droits de l'homme critique dans toute la région, et particulièrement désastreuse en Ouzbékistan et au Turkménistan. Par leur attitude frileuse envers toute autonomie de la "société civile » et leur peur de ne plus contrôler dans sa totalité le fait religieux, les pouvoirs locaux contribuent donc d'eux-mêmes à susciter des mécontentements et accélèrent l'ancrage social d'un islam alternatif, que celui-ci soit appelé à se radicaliser ou non.

L'Asie centrale dans son ensemble répond donc à un modèle d'autoritarisme postsoviétique que l'on retrouve par exemple dans la Biélorussie d'Alexandre Loukachenko, bien plus qu'à un schéma inspiré de la Turquie ou de l'Iran: les liens culturels et linguistiques avec ces pays ainsi que leur appartenance commune au monde musulman ne doivent pas effacer l'histoire des deux derniers siècles, faisant de l'Asie centrale un espace profondément russifié et soviétisé dans ses modes de fonctionnement et dans ses cadres conceptuels. Les chefs d'État ont d'autant plus cherché à développer ce modèle d'autoritarisme laïc présenté comme "éclairé » que, depuis l'intervention américaine en Afghanistan, la région met en avant son rôle de rempart face à l'islamisme, ce qui permet aux pouvoirs en place de jouer la carte du chantage à l'islamisme en cas d'effondrement de leur régime, tout en renforçant à outrance leur autoritarisme.

L'alliance entre États-Unis et Ouzbékistan, avantageuse pour les deux partenaires sur le plan stratégique et militaire ${ }^{25}$, dépend alors en grande partie du choix fait par Washington face à la spirale répressive de Tachkent. Une condamnation plus ferme de celle-ci, comme après le 13 mai, pourrait conduire l'Ouzbékistan à s'impliquer davantage dans l'Organisation de coopération de Shanghai ${ }^{26}$. Celle-ci présente l'intérêt de ne pas s'immiscer dans les affaires politiques de ses membres, la Chine et la Russie soutenant même la politique répressive menée par Islam Karimov au nom de la stabilité contre le risque islamiste. Éluder la question politique permettra aux États-Unis de garder à leurs côtés le pays le plus anti-russe de l'Asie centrale et de s'y installer durablement. Cela suppose toutefois de postuler que, sur le long terme, l'appauvrissement de la population et l'impossibilité d'une quelconque contestation politique ne mèneront pas à l'effondrement, sous une forme ou une autre, du gouvernement ouzbek actuel. Les événements d'Andijan, en effet, portent à s'interroger sur la capacité de survie du régime.

Ainsi, «l'obstacle à la démocratisation ne provient pas tant des islamistes centristes que d'élites laïques conservatrices soucieuses avant tout de verrouiller leur pouvoir et d'exclure les islamistes. $»^{27}$ L'Ouzbékistan, comme ses voisins, profite en effet du contexte international de peur de l'islamisme pour se refermer. Les révolutions géorgienne, ukrainienne et kirghize sont comprises comme une nouvelle attaque, cette fois-ci directe, des Occidentaux et tout particulièrement des États-Unis, afin d'isoler la Russie, afin de permettre l'apparition sur les marges de l'espace post-soviétique de « régimes frères » pro-occidentaux. Depuis 2004, les pays d'Asie centrale resserrent en conséquence leurs liens avec la Russie, tentent de faire bloc face à cette ingérence et raidissent très nettement leur attitude envers les ONG présentes dans leur république. Le rétrécissement de l'espace public, déjà visible depuis plusieurs années en Ouzbékistan, semble aujourd'hui atteindre le Kazakhstan et le Tadjikistan. Il est dès lors probable que des évolutions de fond, si elles se confirment à court ou moyen terme, 
seront gérées par de «nouvelles » équipes au pouvoir. Après le maillon le plus faible, le Kirghizstan, la vague de "révolutions " en cours dans l'ancien espace soviétique pourrait bien, en effet, atteindre les autres Républiques de la région, même si les pouvoirs en place affirment pour l'instant être résolus à ne pas céder aussi facilement.

\section{NOTES}

1. M. Laruelle, "Mondialisation et alter-mondialisme dans les réflexions des milieux politiques et intellectuels d'Asie centrale », La Pensée, Paris, n³ 338, 2004, pp. 27-36.

2. J. Radvanyi (dir.), Les États postsoviétiques. Identités en construction, transformations politiques, trajectoires économiques, Paris, Armand Colin, 2003, $235 \mathrm{p}$.

3. Pour un état des lieux de l'islam post-soviétique, voir M. Laruelle et S. Peyrouse (dir.), Islam et politique en ex-URSS (Russie d'Europe et Asie centrale), L'Harmattan-IFÉAC, 2005, 338 p.

4. Sur le legs soviétique en Asie centrale, consulter le numéro spécial consacré à cette question de la publication de l'IFÉAC, S. Peyrouse (dir.), Gestion de l'indépendance et legs soviétique en Asie centrale. Cahiers d'Asie centrale, n 13-14, 2004, IFÉAC-Édisud, $334 \mathrm{p}$.

5. Les chiffres officiels sont d'environ 150 morts, ceux des milieux associatifs locaux et des témoins de plus de 500. Il faut également noter que près de 500 personnes ont franchi la frontière avec le Kirghizstan et sont aujourd'hui dans une situation juridique et matérielle extrêmement précaire. Installés dans un camp de fortune géré par le hor, ils ne peuvent revenir en Ouzbékistan sous peine de se voir accusés d'avoir participé aux " actes terroristes » du 13 mai.

6. Tous les États d'Asie centrale comptent d'importantes minorités nationales, mais les «nouveaux » pouvoirs sont largement dominés, voire monopolisés par les éponymes, Ouzbeks en Ouzbékistan, Tadjiks au Tadjikistan, Kazakhs au Kazakhstan, etc.

7. A. Akaev, «Stav prezidentom â poterâl vse stepeni svobody » [Devenu président, j’ai perdu toute liberté], Ekspress K., 6 juillet 1993, p. 9.

8. I. Karimov, Uzbekistan na poroge xxi veka. Ugrozy bezopasnosti, usloviâ i garantii progressa [L'Ouzbékistan au seuil du xxie siècle. Menaces à la sécurité, conditions et garanties du progrès], Moscou, Drofa, 1997, p. 131.

9. Sur la question chrétienne en Asie centrale, voir S. Peyrouse, Des chrétiens entre athéisme et islam: regards sur la question religieuse en Asie centrale soviétique et post-soviétique, Paris, Maisonneuve \& Larose, 2004.

10. Article 1 de la Constitution du Kazakhstan de 1995 et de celle du Turkménistan, article 1 et 100 de la Constitution tadjike, article 1.1 de la Constitution kirghize.

11. Article 58 de la Constitution kazakhe, article 8 de la Constitution kirghize, article 8 de la Constitution tadjike, article 11 de la Constitution turkmène.

12. Article 31 de la Constitution ouzbèke, article 16 de la Constitution kirghize, article 26 de la Constitution tadjike, article 11 de la Constitution turkmène.

13. Article 18 de la Constitution ouzbèke.

14. Article 19 de la Constitution kazakhe, article 15 de la première Constitution kirghize, article 17 de la Constitution turkmène.

15. C'est par exemple le cas du Kirghizstan, où le Conseil aux affaires religieuses a été remplacé par une Commission d'État aux affaires religieuses. 
16. E. Abdullaev, «The Central Asian Nexus : Islam and Politics », in B. Rumer (dir.) Central Asia : a Gathering Storm? Armonk, New-York, M. E. Sharpe, 2002, p. 256.

17. S. Peyrouse, «Le christianisme en Asie centrale : miroir des évolutions politiques ", Religions, pouvoir et société, Europe centrale, Balkans, cei. Le courrier des pays de l'Est, Paris, La Documentation française, 2004, $\mathrm{n}^{\circ}$ 1045, pp. 51-61.

18. Sur la situation jugée favorable et les bonnes relations entre l'archevêque d'Asie centrale Vladimir Ikim et les différents pouvoirs, voir par exemple Slovo zhizni, 14 août 1995, n 5 (117), p. $1 ; 20$ septembre $1995, \mathrm{n}^{\circ} 6$ (118), pp. 1 et 3. L'idée de respect des droits religieux nous a été systématiquement confirmée par tous les représentants de l'Église orthodoxe que nous avons rencontrés.

19. Sur la guerre civile au Tadjikistan, voir le dossier S. Dudoignon (dir.), « Le Tadjikistan existet-il ? Destins politiques d'une "nation imparfaite" ", Cahiers d'études sur la Méditerranée orientale et le monde turco-iranien (CÉMOTI), Paris, CÉRI, $\mathrm{n}^{\circ}$ 18, 1994.

20. Voir, entre autres, "Said Abdullo Nuri: “my hotim sodejstvovat' politicheskomu, èkonomicheskomu, social'nomu i kul'turnomu razvitiû Tadzhikistana" ", [Said Abdullo Nuri : «Nous voulons contribuer au développement politique, économique, social et culturel du Tadjikistan »] Biznes i Politika, n² 2 (470), 11 janvier 2002, p. 2.

21. B. Babadjanov, "Le renouveau des communautés soufies en Ouzbékistan ", Cahiers d'Asie centrale, $n^{\circ}$ 5-6, 1998, pp. 285-300.

22. M. B. Olcott et B. Babadjanov, «The Terrorist Notebooks », Foreign Policy, mars-avril 2003, pp. 31-40.

23. B. Babadjanov, «Islam officiel contre islam politique en Ouzbékistan aujourd'hui: la Direction des musulmans et les groupes non-hanafî ", Revue d'études comparatives Est-Ouest, vol. 31, n³, 2000, pp. 151-164; du même auteur: "Ferganskaâ dolina: istochnik ili zhertva islamskogo fundamentalizma? » [La vallée du Ferghana : source ou victime du fondamentalisme islamiste ?], Central'naâ Aziâ i Kavkaz, n 4(5), 1999, pp. 125-131.

24. A. K. Zajfert et I. D. Zvâgel'skaâ, « Primirenie Evropy i islama v Evrazii » [La réconciliation de l'Europe et de l'islam en Eurasie], Vostok/Oriens, n 5, 2004, pp. 76-90. Pour un commentaire critique de cet article, voir B. Babadjanov et M. B. Olcott, « Sécularisme et islam politique en Asie centrale ", in M. Laruelle et S. Peyrouse (dir.), Islam et politique en ex-urss (Russie d'Europe et Asie centrale), op. cit., pp. 323-336.

25. Cette coopération s'est particulièrement renforcée lors des opérations militaires américaines en Afghanistan. Les États-Unis se sont installés dès l'automne 2001 au sud de la ville de Karchi (Khanabad) en Ouzbékistan, ouvrant alors - fait historique majeur - leur première base militaire sur le territoire ex-soviétique. Ils ont par ailleurs obtenu une seconde base militaire à Bichkek (Kirghizstan) et ont négocié avec le Tadjikistan afin de pouvoir utiliser les bases gouvernementales, sans envoi de troupes américaines. L'Ouzbékistan se démarque de ses voisins par son soutien systématique aux choix américains et a soutenu l'intervention en Afghanistan tout comme celle en Irak.

26. Composée de la Russie, la Chine, l'Ouzbékistan, le Kazakhstan, le Kirghizstan et le Tadjikistan, cette organisation, créée en 1996, tente de renforcer les liens économiques et politiques entre les pays membres. L'Organisation de coopération de Shanghai s'est particulièrement orientée depuis plusieurs années vers une lutte commune contre le terrorisme (auquel sont associés le séparatisme ouigour et toutes les tendances salafistes de l'islam), le trafic de drogue et d'armes, afin de "garantir la stabilité de la région " et surtout d'empêcher une implantation trop directe des États-Unis au cœur de ladite « Eurasie ».

27. O. Roy, L'islam mondialisé, Paris, Seuil, nouvelle édition, 2004, p. 46. 


\section{RÉSUMÉS}

La « révolution» kirghize de mars 2005 marque l'entrée probable de l'Asie centrale dans une phase d'instabilité politique. En Ouzbékistan, la répression des manifestations de mai 2005 n'a fait qu'affaiblir la légitimité d'un pouvoir qui, en éliminant toute opposition laïque, s'est enfermé dans un face-à-face avec les islamistes. Le «renouveau " vanté par les régimes en place après 1991 concédait à l'islam une place nouvelle mais très contrôlée, et donnait un tour particulier à la question de la réforme dans des pays de tradition musulmane officiellement laïcs. Les rapports qu'entretiennent l'État post-soviétique et l'islam officiel, et la capacité de cet islam institutionnel à réguler les revendications discrètes de la population, détermineront les conditions du succès ou de l'échec de la minorité islamiste en Asie centrale.

The "revolution" in Kyrgyzstan in March 2005 may signal the entry of Central Asia into a period of political instability. In Uzbekistan, the repression of demonstrations in May 2005 further eroded the legitimacy of a regime that, by eliminating all secular opposition, is now locked in confrontation with Islamists. The "renewal" lauded by those in power after 1991 offered Islam an improved but tightly controlled status, and reshaped the question of reform in those countries of Muslim tradition that are officially defined as "secular". The relationship between the postSoviet State and official Islam, and the capacity of this institutionalised Islam to mediate between the differing claims of the population, will largely determine the success or failure of the Islamist minority in Central Asia.

\section{INDEX}

Keywords : Central Asia, Islam, Islamism, Politics, Religion, Secularism, ussr, Uzbekistan Mots-clés : Asie centrale, islam, islamisme, laïcité, Ouzbékistan, politique, religion, urss

\section{AUTEUR}

\section{SÉBASTIEN PEYROUSE}

Institut français d'études sur l'Asie centrale (ifÉac), Tachkent.

Docteur de l'Institut national des langues et civilisations orientales (Paris), Sébastien Peyrouse est chercheur à l'Institut français d'études sur l'Asie centrale (Tachkent). Il travaille sur les rapports entre religion et politique ainsi que sur la question des minorités nationales dans l'Asie centrale contemporaine. Il a publié : Des chrétiens entre athéisme et islam. Regards sur la question religieuse en Asie centrale soviétique et postsoviétique (Paris, Maisonneuve \& Larose, 2003) et Les Russes du Kazakhstan. Identités nationales et nouveaux États dans l'espace post-soviétique (avec Marlène Laruelle, Maisonneuve \& Larose, 2004). Il a aussi dirigé deux ouvrages : Islam et politique en exURSS (Russie d'Europe et Asie centrale) (avec Marlène Laruelle, Paris, L'Harmattan, 2005) et Gestion de l'indépendance et legs soviétique en Asie centrale, numéro des Cahiers d'Asie centrale (IFÉAC-Édisud. n 13-14, 2004).

sebpeyrouse@yahoo.com 